\title{
Environmental Objectives, Cost Efficiency, and Multivariate Stochastic Control*
}

\author{
Chuan-Zhong $\mathrm{Li}^{1}$ and Erika Budh${ }^{2}$ \\ ${ }^{1}$ Department of Economics, Uppsala University, and Department of \\ Economics and Society, Dalarna University, Sweden \\ ${ }^{2}$ The Swedish Environmental Protection Agency, Stockholm, Sweden
}

\begin{abstract}
This paper is concerned with the cost efficiency in achieving the Swedish national air quality objectives under uncertainty. To realize an ecologically sustainable society, the parliament has approved a set of interim and longterm pollution reduction targets. However, there are considerable quantification uncertainties on the effectiveness of the proposed pollution reduction measures. In this paper, we develop a multivariate stochastic control framework to deal with the cost efficiency problem with multiple pollutants. Based on the cost and technological data collected by several national authorities, we explore the implications of alternative probabilistic constraints. It is found that a composite probabilistic constraint induces considerably lower abatement cost than separable probabilistic restrictions. The trend is reinforced by the presence of positive correlations between reductions in the multiple pollutants.
\end{abstract}

Key words: Environmental Objectives, Cost Efficiency, and Multivariate Stochastic Control

\section{Introduction}

The vision for Swedish environmental policy is to realize an ecologically sustainable society within a generation's time (cf Ministry of the Environment, 2004). This

*This research is financed by the Swedish Environmental Protection Agency. The authors thank Lars Hultkrantz, Karl-Gustaf Löfgren and Runar Brännlund for valuable comments and suggestions. The usual disclaimer applies. 
vision has been specified in the fifteen adopted national environmental quality objectives, which among others include, clean air, reduced climate impact, and a non-toxic living environment (SGB 1997/98:145). Moreover, the government has set a number of interim targets for the year 2010, and proposed potential measures for compliance (SGB 2000/01:130). This paper is concerned with a subset of these interim targets. This paper is concerned with a subset of these interim targets, namely, the reduction in air pollutants such as nitrogen oxide $\left(\mathrm{NO}_{x}\right)$, sulphur dioxide $\left(\mathrm{SO}_{2}\right)$, volatile organic compounds (VOC), particulate matters and carbon dioxide $\left(\mathrm{CO}_{2}\right)$. Since the various pollution reduction measures differ greatly in both cost and effectiveness, the problem in concern is to find the least cost solution for achieving a particular set of targets.

The cost efficiency problem was first studied by the Committee of Environmental Objectives (SGOR 2000:52) in a total-cost-framework. In a subsequent study using linear programming models, Budh (2004a) finds that marginal costs could be zero for certain pollutants with overshoot targets parallel to the satisfaction of other objectives. Nevertheless the main conclusion from this deterministic model is that there are no important losses associated with a separate emission by emission approach. However, both of the studies ignore the quantification uncertainties involved in the effectiveness of the emission reduction measures. In this paper, we explicitly take such uncertainties into account in our cost efficiency analysis of the environmental objectives.

The basic module is the chance-constrained programming model (Charnes and Cooper 1964), which minimizes the total abatement cost under one or more probabilistic constraints (Beavis and Walker 1983, McSweeny and Shortle, 1990; Elofsson 2000, Gren et al 2000, Budh 2004b). The contribution in this paper is that we allow for composite, integrated probabilistic constraints, with possible correlation structures on the reduction of multiple pollutants. The estimation of such a model is made computationally feasible by using GAUSS where procedures for various probability distributions such as the multivariate normal one are available.

The remaining part of the paper is structured as follows. Section 2 formulates the basic stochastic programming model with separable probabilistic constraints, and section 3 describes the data and presents the results from the basic model. In section 4 , we develop the model further to accommodate for the multivariate nature of the problem, and compare these results with those from the basic model. Section 5 sums up the study. 


\section{The Basic Stochastic Control Model}

Consider a number of $n$ measures for pollution control. Let $x_{j}$ denote a control variable with a value 1.0 if measure $j$ is undertaken, and 0 else, where $j=1,2, \ldots, n$. We also allow for interior values of $x_{j}$ in the interval $[0,1]$ to reflect partial implementation. Let the expected cost of measure $j$, if fully implemented, be $c_{j}$, then the total expected cost associated with any feasible set of control $\left\{x_{j}, j=1,2, \ldots, n\right\}$ can be described by

$$
C=\sum_{j=1}^{n} c_{j} x_{j}
$$

Consider that there are $m<n$ pollutants to be controlled. Let $a_{i j}$ denote the technological parameters representing the reduction in the emission of pollutant $i$ if measure $j$ is fully implemented, with $i=1,2, \ldots, m$, and $j=1,2, \ldots, n$. Then the emission reduction in the $i$ :th pollutant can be expressed as

$$
R_{i}=\sum_{j=1}^{n} a_{i j} x_{j}, i=1,2, \ldots, m
$$

Since the technological parameters are not known with certainty, we need to model them as stochastic variables. As a first attempt, we assume that each $a_{i j}$ is normally and independently distributed with mean $\bar{a}_{i j}$ and variance $v_{i j}$. Then, the actual emission reduction in pollutant $i$ will also be normally and independently distributed with mean $\bar{R}_{i}=\sum_{j=1}^{n} \bar{a}_{i j} x_{j}$ and variance $V_{i}=\sum_{j=1}^{n} v_{i j} x_{j}^{2}$. For a given set of pollution reduction targets $\left\{T_{i}: i=1,2, \ldots, m\right\}$, the probability for each target to be satisfied can be described by

$$
\operatorname{Pr}\left(R_{i} \geq T_{i}\right)=\Phi\left(\frac{\sum_{j=1}^{n} \bar{a}_{i j} x_{j}-T_{i}}{\sigma_{i}}\right)
$$

where $\sigma_{i}=\sqrt{V_{i}}$ is the standard error of $R_{i}, i=1,2, \ldots, m$. Let $\alpha_{i}$ denote the required minimum probability level for target $i$ to be reached, then the stochastic constraints for the $m$ targets can be expressed as

$$
\Phi\left(\frac{\sum_{j=1}^{n} \bar{a}_{i j} x_{j}-T_{i}}{\sigma_{i}}\right) \geq \alpha_{i}, i=1,2, \ldots, m
$$

The basic stochastic control model can now be formulated as minimizing the expected abatement cost in (1) under the $m$ separable probabilistic constraints in 
(4). For any feasible set of control $\left\{x_{j}: j=1,2, \ldots, n\right\}$, the Lagrangian becomes

$$
L=\sum_{j=1}^{n} c_{j} x_{j}+\sum_{i=1}^{m} \lambda_{i}\left[\alpha_{i}-\Phi\left(\frac{\sum_{j=1}^{n} \bar{a}_{i j} x_{j}-T_{i}}{\sigma_{i}}\right)\right]
$$

where $\lambda_{i}$ are the Lagrangian multipliers associated with the probability constraints. From the properties of the normal distribution, it can be shown that, for $\alpha=0.5$, the solution to (5) would be the same as to the problem with deterministic constraints $\sum_{j=1}^{n} \bar{a}_{i j} x_{j} \geq T_{i}, i=1,2, \ldots, m$, meaning that uncertainty would not have any effect on the cost minimizing solution (cf. Beavis and Walker, 1983; Gren et al., 2000). For $\alpha_{i}>0.5$, more stringent controls will be necessary for compliance such that the expected pollution reduction on each target $i$ should be larger than the target itself such that $\bar{R}_{i}>T_{i}$.

In this paper, we only consider the case with $\alpha_{i}$ values greater than 0.5 , with which the probability function in (4) is strictly concave in the control variables. This implies that the feasible set $\left\{x_{j}, j=1,2, \ldots, n\right\}$ is strictly convex set, and thus minimizing the Lagrangian in (5) leads to a unique cost-efficient solution $\left\{x_{j}^{*}\right\}$. The total pollution abatement cost can thus be expressed by $C^{*}=\sum_{j=1}^{n} c_{j} x_{j}^{*}$, and the marginal cost with respect to the pollution reduction target $T_{i}$, can be derived to be

$$
M C_{i}=-\lambda_{i}^{*} \frac{\partial \Phi_{i}^{*}}{\partial T_{i}}, i=1,2, \ldots, m
$$

where $\lambda_{i}^{*}$ is the optimal value of the Lagrangian multiplier, and $\Phi_{i}^{*}$ denotes the cumulative probability distribution function in (3) concerning target $i$, evaluated at the optimal control values $\left\{x_{j}^{*}\right\}$. The marginal cost $M C_{i}$ defined in this way corresponds to the cost incurred from increasing the pollution reduction target $T_{i}$ by an extra unit. Since $\lambda_{i}^{*}=\partial C^{*} / \partial \alpha_{i} \geq 0$, and $-\partial \Phi_{i}^{*} / \partial T_{i}>0$, it is obvious that $M C_{i} \geq 0$, and thereby the marginal cost $M C_{i}$ is non-decreasing in the target $T_{i}$.

\section{Data and the Basic Results}

The data used in this study were collected by several national authorities (SGOR 2000:23, SGOR 2002:52). In this study, we focus on five different air pollutants, namely nitrogen oxide (NOX), sulphur dioxide (SO2), carbon dioxide (CO2), particulate matters and volatile organic compounds (VOC). To reduce the emission of these pollutants, 85 measures have been proposed, each is defined as an action taken by households, firms or the public sector. These include actions such as energy savings, technological efficiency improvements, technical abatements and structural 
changes (cf Sternhufvud and Grennfelt, 2001). The annualized cost $c_{j}$, the expected value $\bar{a}_{i j}$ and the variance $v_{i j}$ of the abatement effects associated with each measure $j$ are reported in the Appendix, where $i=1,2, \ldots, 5$ and $j=1,2, \ldots, 85$. It can be seen that some measures involve a negative cost. The reason for this is that these measures, which are economically profitable from a production point of view, would be undertaken anyway even without the air quality objectives ${ }^{1}$. Although the initial investment would cost a considerable amount, the economic benefit it generates in the future would more than recover the investment cost for these measures. As shown in Budh (2004a), the baseline reduction in the emission of carbon dioxide is not zero but 1979 thousands tons due to the productivity enhancing measures. Since CO2 cannot be reduced by technical abatement, these measures target the fuel consumption and thereby give a financial saving. However, only financial costs are included in our data. Social costs in time or comfort are thus excluded, but may be important for some of the measures.

The governmental interim pollution reduction targets $T_{i}$ are formulated with three different ambition levels, labelled as "low", "middle" and "high". Without loss of generality, this paper only deals with the "high" ambition level as shown along the second column in Table 1. By choosing the least compliance probability level for each of the 5 pollutants as $\alpha_{i}=0.95$, we solve the cost minimization problem (1) subject to the probabilistic constraints (4) using a modified version ${ }^{2}$ of the "constrained optimization" procedure in GAUSS. The advantage in using GAUSS is that the many cumulative distributions functions are readily available without any need to convert the probabilistic constraint to deterministic equivalents. This is especially useful for stochastic multivariate control where it may be impossible to convert an integrated probabilistic constraint to a determinant equivalent counterpart. For the basic model above, we summarize the results in Table 1.

Consistent with the theoretical predictions, the expected values of pollution reduction exceed the target levels in order to reach the lower bound of compliance probabilities greater than 0.5 . The actual compliance probabilities are exactly 0.95 , indicating that the constraints for $\mathrm{NO}_{x}, \mathrm{SO}_{2}, \mathrm{VOC}$ and $\mathrm{CO}_{2}$, are all binding. The marginal costs for these pollutants are thus all positive. For the suspended particular matters, the actual compliance probability exceeds 0.95 and thus the marginal cost is zero. When the other four targets are reached by an optimal set of measures, the

\footnotetext{
${ }^{1}$ It is worth mentioning that the presence of negative costs seems to lead to rather low total abatement cost for reaching the environmental objectives. Fortunately, this paper concerns more with the relative performances of different probabilistic constraints rather than the absolute amount of the total cost.

${ }^{2}$ Since the original version of "constrained optimization" only allows a nonlinear cost function, we have to minimize a monotonous transformation of our linear cost function.
} 
Table 1: Cost efficient solutions from the basic model

\begin{tabular}{lrrrr}
\hline Pollutant & $\begin{array}{r}\text { Target } \\
\text { (ton) }\end{array}$ & $\begin{array}{r}\text { Expected } \\
\text { reduction }\end{array}$ & $\begin{array}{r}\text { Probability of } \\
\text { compliance }\end{array}$ & $\begin{array}{r}\text { Marginal cost } \\
(\mathrm{SEK} / \mathrm{kg})\end{array}$ \\
\hline $\mathrm{NO}_{x}$ & 23000 & 24082 & 0.9500 & 31.35 \\
$\mathrm{SO}_{2}$ & 8000 & 8459 & 0.9500 & 35.83 \\
$\mathrm{VOC}$ & 85000 & 91310 & 0.9500 & 450.82 \\
Particles & 10000 & 18535 & 0.9996 & 0.00 \\
$\mathrm{CO}_{2}$ & 4890000 & 5103000 & 0.9500 & 0.48 \\
\hline \multicolumn{2}{l}{ The total abatement cost $C^{*}=$} & SEK 1598.70 millions
\end{tabular}

target for this pollutant is automatically satisfied at a higher probability level than necessary so that a marginal increase in the target does not lead to any extra cost. The measures that primarily target VOC also reduce particle emissions substantially (Budh 2004a). In addition to the marginal costs, it would also be interesting to trace the total cost for comparison purposes across different models. The minimized total cost for the basic model above turns out to be SEK $1.5987 \times 10^{9}$, about 1600 million Swedish Crowns yearly.

\section{The Multivariate Stochastic Control Model and Results}

Note that the probabilistic constrains in the previous sections are formulated separately for each of the pollution reduction targets. This section attempts to develop a multivariate stochastic control model by introducing a joint probability constraint with possible correlation structures. The new model here has the advantage of allowing internal trade-offs between the compliance probabilities in fulfilling the different targets, and thus may induce a lower total abatement cost compared to the separable control model. Hence, it can better handle all effects of given measures in reducing multiple pollutants.

Let $\mathbf{R}=\left(R_{1}, R_{2}, \ldots, R_{m}\right)^{\prime}$ denote the m-dimensional column vector of stochastic pollution reductions with $R_{i}=\sum_{j=1}^{n} a_{i j} x_{j}, i=1,2, \ldots, m$, and $\mathbf{T}=\left(T_{1}, T_{2}, \ldots, T_{m}\right)^{\prime}$ the corresponding vector of preset targets. Then, the joint probabilistic constraint in its most general form can be formulated as

$$
\operatorname{Pr}(\mathbf{R} \geq \mathbf{T}) \geq \alpha
$$

where $\operatorname{Pr}(\mathbf{R} \geq \mathbf{T})=\operatorname{Pr}\left(R_{1} \geq T_{1}, R_{2} \geq T_{2}, \ldots R_{m} \geq T_{m}\right)$ by definition, and $\alpha$ is the scalar, minimum probability level for the $\mathrm{m}$ targets to be jointly satisfied. Since $a_{i j}$ 
has a mean value $\bar{a}_{i j}$ for each given $i$ and $j$, the mean abatement effect on pollutant $i$ becomes $\bar{R}_{i}=\sum_{j=1}^{n} \bar{a}_{i j} x_{j}$, for $i=1,2, \ldots, m$. Let $\overline{\mathbf{R}}=\left(\bar{R}_{1}, \bar{R}_{2}, \ldots, \bar{R}_{m}\right)^{\prime}$ denote the mean of the vector $\mathbf{R}$, then we can express $\mathbf{R}$ by

$$
\mathbf{R}=\overline{\mathbf{R}}-\varepsilon
$$

where $\varepsilon=\left(\varepsilon_{1}, \varepsilon_{2}, . ., \varepsilon_{m}\right)^{\prime}$ is the vector of stochastic disturbances with $\varepsilon_{i}=\bar{R}_{i}-R_{i}$ for each $i=1,2, \ldots, m$.

The joint probability on the left-hand-side of (7) can now be written as

$$
\operatorname{Pr}(\mathbf{R} \geq \mathbf{T})=\operatorname{Pr}(\overline{\mathbf{R}}-\varepsilon \geq \mathbf{T})=\operatorname{Pr}(\varepsilon \leq \overline{\mathbf{R}}-\mathbf{T})
$$

Assume that the stochastic disturbances $\varepsilon$ be normally distributed with zero means and a variance-covariance matrix $\mathbf{S}$, then the probability expression in (9) can be more precisely expressed as

$$
\begin{aligned}
\operatorname{Pr}(\mathbf{R} \geq \mathbf{T})= & \Phi(\overline{\mathbf{R}}-\mathbf{T}, \mathbf{S}) \\
\equiv & \int_{-\infty}^{\overline{\mathbf{R}}-\mathbf{T}}(2 \pi)^{-\frac{m}{2}}|\mathbf{S}|^{-\frac{1}{2}} \exp \left[-\frac{1}{2} \boldsymbol{\varepsilon}^{\prime} \mathbf{S}^{-1} \boldsymbol{\varepsilon}\right] d \boldsymbol{\varepsilon}
\end{aligned}
$$

(cf Greene, 2002). The cost efficiency problem can now be formulated as minimizing (1) subject to the following joint probability constraint

$$
\Phi(\overline{\mathbf{R}}-\mathbf{T}, \mathbf{S}) \geq \alpha
$$

The Lagrangian become

$$
L=\mathbf{c}^{\prime} \mathbf{x}+\lambda[\alpha-\Phi(\overline{\mathbf{R}}-\mathbf{T}, \mathbf{S})]
$$

where $\mathbf{c}=\left(c_{1}, c_{2}, \ldots, c_{n}\right)^{\prime}$ denotes the cost vector, $\mathbf{x}=\left(x_{1}, x_{2}, \ldots, x_{n}\right)^{\prime}$ the control vector satisfying $x_{j} \in[0,1]$ for each $j=1,2, \ldots, n$, and $\lambda$ the Lagrange multiplier associated with the probability constraint in (11). By minimizing $L$ in (12) for given combinations of $(\mathbf{T}, \alpha)$, we can obtain an optimal solution $\mathbf{x}^{*}=\left(x_{1}^{*}, x_{2}^{*}, \ldots, x_{n}^{*}\right)$ for the control variables and $\lambda^{*}$ for the auxiliary variable. The minimized abatement cost then becomes $C^{*}=\mathbf{c}^{\prime} \mathbf{x}^{*}$, and the marginal cost with respect to the target vector can be expressed by

$$
\mathbf{M C}^{*}=-\lambda^{*} \frac{\partial \Phi(\overline{\mathbf{R}}-\mathbf{T}, \mathbf{S})}{\partial \mathbf{T}}
$$

Note that there is an essential difference between the marginal cost in equation (6) and that in (13). While the former is defined separately for each probability 
Table 2: Results from a non-correlated joint probability model

\begin{tabular}{lrrrr}
\hline Pollutant & $\begin{array}{r}\text { Target } \\
\text { (ton) }\end{array}$ & $\begin{array}{r}\text { Expected } \\
\text { reduction }\end{array}$ & $\begin{array}{r}\text { Probability of } \\
\text { compliance }\end{array}$ & $\begin{array}{r}\text { Marginal cost } \\
(\mathrm{SEK} / \mathrm{kg})\end{array}$ \\
\hline $\mathrm{NO}_{x}$ & 23000 & 24681 & 0.9976 & 51.01 \\
$\mathrm{SO}_{2}$ & 8000 & 8844 & 0.9993 & 38.89 \\
$\mathrm{VOC}$ & 85000 & 87904 & 0.7806 & 406.34 \\
Particles & 10000 & 18865 & 0.9998 & 1.33 \\
$\mathrm{CO}_{2}$ & 4890000 & 5224369 & 0.9946 & 0.48 \\
\hline \multicolumn{2}{l}{ The total abatement cost $C^{*}=$} & SEK 299.96 millions \\
\hline
\end{tabular}

constraint $\operatorname{Pr}\left(R_{i}>T_{i}\right)$, the latter is based on one and the same joint probability distribution with interdependent targets. We solve the problem (12) using the "constrained optimization" procedure embedded in GAUSS where the multivariate normal distribution function is evaluated with the cdfmvn command. To be comparable with the separable constraint model, we consider the same "high" ambition target vector as before, and take the minimum joint probability level $\alpha=\prod_{i=1}^{m} \alpha_{i}$. Since $m=5$ and $\alpha_{i}=0.95$ for each $i(i=1,2, \ldots, 5)$ in our application, we should have $\alpha=0.95^{5} \approx 0.77$.

After having set a comparable compliance probability level $\alpha$ for the same targets, we have to specify the variance-covariance matrix $\mathbf{S}$. In a first attempt, we assume no correlation between reductions of the different pollutants. In this case, the main diagonal of $\mathbf{S}$ would contain the individual variances $\sigma_{i}^{2}$ for $i=1,2, \ldots, 5$ with zero values for all other elements. With these parameters, we obtain the results as shown in Table 2. Compared to the results from the separable constraint model in Table 1, we find that the ex-post compliance probability for VOC is much lower with the joint probability model whereas the compliance probabilities for the other pollutants are considerably higher. Except for CO2, the associated marginal costs adjust, i.e. increase, with the higher probabilities for compliance. Hence the new model implicitly optimizes the ex-post compliance probabilities by making internal trade-offs between the expected pollution reduction in different pollutants $\bar{R}_{i}$. If it is more expensive to reduce a unit of pollutant $i$, then it would be more cost efficient to satisfy the condition $\alpha=\prod_{i=1}^{m} \alpha_{i}$ by lowering the $\bar{R}_{i}$ and thereby $\alpha_{i}$ in exchange for increase in $\bar{R}_{k}$ or equivalently $\alpha_{k}$ for $k \neq i$. To fix ideas, one may consider an "as-if" problem of minimizing a cost function $\sum_{i=1}^{m} \lambda_{i} \alpha_{i}$ subject to the constraint $\prod_{i=1}^{m} \alpha_{i}=\alpha$. As depicted in Figure 1, if the marginal cost $\lambda_{i}$ is relatively high compared to any $\lambda_{k}, k=1,2, . . m$, then it would be more efficient to "reallocate" the compliance probabilities from point $A$ with $\alpha_{i}=\alpha_{k}$ to point $B$ with $\alpha_{k}>\alpha_{i}$. At the most efficient allocation point $B$, the iso-cost line is tangent to the iso- 
Table 3: Results from joint probability model with correlation

\begin{tabular}{lrrrr}
\hline Pollutant & $\begin{array}{r}\text { Target } \\
\text { (ton) }\end{array}$ & $\begin{array}{r}\text { Expected } \\
\text { reduction }\end{array}$ & $\begin{array}{r}\text { Probability of } \\
\text { compliance }\end{array}$ & $\begin{array}{r}\text { Marginal cost } \\
(\mathrm{SEK} / \mathrm{kg})\end{array}$ \\
\hline $\mathrm{NO}_{x}$ & 23000 & 24673 & 0.9975 & 51.88 \\
$\mathrm{SO}_{2}$ & 8000 & 8844 & 0.9991 & 39.87 \\
$\mathrm{VOC}$ & 85000 & 87905 & 0.7807 & 404.82 \\
Particles & 10000 & 18736 & 0.9997 & 0.38 \\
$\mathrm{CO}_{2}$ & 4890000 & 5223028 & 0.9945 & 0.49 \\
\hline \multicolumn{2}{l}{ The total abatement cost $C^{*}=$} & SEK 286.26 millions \\
\hline
\end{tabular}

compliance probability curve $\prod_{i=1}^{m} \alpha_{i}=\alpha$. However, if the marginal damage from failure in compliance is extremely high for a specific pollutant, it may be necessary to specify a separate restriction for it to make compliance more likely even though the reduction cost would increase.

By lowering the compliance probability for VOC, the marginal cost per unit pollution reduction declines considerably from SEK 450 to 406 per kilogram. Although the marginal costs for the other pollutants have increased to some extent, the total abatement cost is greatly reduced to SEK $2.9996 \times 10^{8}$, i.e. about 300 millions Swedish Crowns yearly.

Finally, we deal with the more general case with a correlation structure. Let us arrange the standard deviations $\boldsymbol{\sigma}_{i}$ in a matrix form

$$
\mathbf{A}=\left[\begin{array}{cccc}
\sigma_{1} & 0 & 0 & 0 \\
0 & \sigma_{2} & 0 & 0 \\
0 & 0 & \ldots & 0 \\
0 & 0 & 0 & \sigma_{m}
\end{array}\right]
$$

and denote the $m \times m$ correlation matrix by

$$
\mathbf{B}=\left[\begin{array}{cccc}
1 & r_{12} & \ldots & r_{1 m} \\
r_{21} & 1 & . . & r_{2 m} \\
\ldots & \ldots & 1 & \ldots \\
r_{m 1} & r_{m 2} & \ldots & 1
\end{array}\right]
$$


where $r_{i k}=r_{k i}$, then the variance-covariance matrix $\mathbf{S}$ in (11) can be expressed as

$$
\mathbf{S}=\mathbf{A B A}=\left[\begin{array}{cccc}
\sigma_{1}^{2} & r_{12} \sigma_{1} \sigma_{2} & \ldots & r_{1 m} \sigma_{1} \sigma_{m} \\
r_{21} \sigma_{2} \sigma_{1} & \sigma_{2}^{2} & \ldots & r_{2 m} \sigma_{2} \sigma_{m} \\
\ldots & \ldots & \ldots & \ldots \\
r_{m 1} \sigma_{m} \sigma_{1} & r_{m 2} \sigma_{m} \sigma_{2} & \ldots & \sigma_{m}^{2}
\end{array}\right]
$$

In principle, we would be able to optimize the Lagrangian in (12) for any given correlation matrix B. However, such information is not available from the current data set. As an illustration, we only consider a strong correlation between the reductions in $\mathrm{NO}_{x}$ and particles with a correlation coefficient being 0.6 , and examine its implications on the cost structure. The resulting marginal and total costs, among other things, are reported in Table 3. Compared to Table 2 with no correlation at all, we can see that the marginal cost for the particles' target reduces from SEK 1.33 to 0.38 per kilogram. The intuition is that when the $\mathrm{NO}_{x}$ target is reached by a set of measures, the reduction target for particles are more or less satisfied as a by-product.

Since Since many measures reduce more than one pollutant, it would be interesting to assess the correlation structure and integrate it into cost efficiency analyses in future research. For this illustration, we find that the simple correlation structure reduces the total abatement cost from about SEK 300 to about 286 millions. Of course, the exact amount of cost saving would depend on the correlation structure.

\section{Concluding Remarks}

This paper develops a multivariate stochastic control model for cost efficiency analysis of the Swedish national environmental quality objectives. The advantage of the model lies in that it takes into account both the uncertainty in the abatement effects of the proposed measures and the interdependency between the reductions of multiple pollutants. As an illustration, we limit our analysis in this study to five interim reduction targets for emissions to air i.e. nitrogen oxide, sulphur and carbon dioxides, particulate matters and volatile organic compounds. Using the Gaussian procedure for multivariate normal distribution and the "constrained optimization" module, the stochastic control problem can be directly solved for any feasible targets and compliance probability levels.

Based on the cost and technology data on measures aiming at air pollution reductions, we have studied the cost efficiency problem with three different probabilistic constraints. First, we analyze the case with separable probabilistic constraints, each for a certain air pollutant. To achieve the most ambitious interim targets in this 
case, the resulting minimum abatement cost turns out to be about SEK 1600 millions. Except for particles, the probabilistic constraints for all other targets are binding with a strictly positive marginal cost. Second, we analyze the case with an integrated, composite probability constraint without any correlation between the reductions in the multiple pollutants. Since this version of the model allows for internal trade-offs between the ex-post compliance probabilities for the various pollutants, the total abatement cost required becomes considerably lower at about SEK 300 millions. The reason is that the optimization program "punishes" pollutants with higher marginal abatement cost by lowering its expected pollution reduction and thereby also the compliance probability. In exchange, the ex-post compliance probabilities for the other pollutants are increased in order to satisfy the joint probability level of compliance. Finally, we take a look at the more general model both with an integrated probabilistic constraint and a correlation structure. It is shown that if the reductions of several pollutants are positively correlated, for a chosen set of measures, then the marginal abatements cost for one or more of these pollutants tend to be lower. Thus, a positive correlation between the stochastic reduction in different pollutants does contribute to a lower total abatement cost. Compared to the case with no correlation, a simple correlation structure with a correlation coefficient of 0.6 between $\mathrm{NO}_{x}$ and $\mathrm{PM}$, leads to a lowering in the marginal cost from 1.33 to 0.38 for PM, and the total abatement cost reduces to about SEK 286 millions.

It is worth mentioning that the present paper does not attempt to be comprehensive in dealing with all environmental objectives in Sweden. We use the interim reduction targets for emissions to air to illustrate the computational tractability of the multivariate stochastic control model and to demonstrate its advantage over the separable constraints formulations in cost efficiency analysis. In addition, we only use a static one-shot cost minimization criterion for the future environmental objectives without any Bayesian type of information feedback. To incorporate the realized effectiveness of the undertaken measures, the availability of new measures, and learning-by-doing experiences in a dynamic optimization framework should be a promising area of future research in cost efficiency analysis of environmental objectives. Obviously, this would require new analytical tools and heavier data inputs.

\section{References}

Beavis, B. and Walker, M., 1983. Achieving environmental standards with stochastic dischanges. Journal of Environmental Economics and Management 10, 103-111. Budh, E., 2004a.Could integrated control enhance efficiency in compliance with 
multiple emission reduction targets? Marginal abatement costs with integrated and separate targets. Manuscript.

Budh, E., 2004b.Will the European CO2 emissions trading scheme succeed? Evidence from Sweden. Manuscript.

Charnes, A. and Cooper, W.W., 1964. Chance-constrained programming. Operations Research 11, 18-39.

Elofsson K, 2000. Cost efficient reductions of stochastic nutrient loads to the Baltic sea. Swedish University of Agricultural Sciences, Department of economics, Working paper 2000:6.

Gauss Applications, 2002. Constrained optimization. Aptech Systems, Inc, Maple Valley, WA, USA.

Greene, W. H., 2002. Econometric analysis (5:th edition). Prentice Hall.

Gren I-M, Destouni G, Scharin H., 2000. Cost effective management of stochastic coastal water pollution. Environmental Modelling and Assessment 5, 193-203.

McSweeny W.T. and Shortle J.S., 1990. Probabilistic cost effectiveness in agricultural nonpoint pollution control. Southern Journal of Agricultural Economics $22,95-104$.

Ministry of the Environment, 2004. Sweden's Environmental Policy. A brief overview. Ref. no. M 2004.03.

SGB, 1997/98:145. Swedish Environmental Quality Objectives. The Swedish Ministry of the Environment, Stockholm.

SGB, 2000/01:130. Swedish environmental objectives - intermediate goals and action plans. The Swedish Ministry of the Environment, Stockholm.

SGOR, 2000:23. Proposed Swedish Climate Strategy. Final report of the Climate Committee, Stockholm.

SGOR, 2000:52. The future environment - our common responsibility. Final report of the Committee on Environmental Objectives, Stockholm.

Sternhufvud C. and Grennfelt, P., 2001. A comparison between the cost curves in the RAINS-model and the Swedish environmental quality objectives. IVL Swedish Environmental Research Institute, Report B1421. 